\title{
Kinetic Compensation Effect in Logistic Distributed Activation Energy Model for Lignocellulosic Biomass Pyrolysis
}

Di Xu ${ }^{1}$, Meiyun Chai ${ }^{1}$, Zhujun Dong ${ }^{1}$, Md. Maksudur Rahman ${ }^{1}$, Xi Yu ${ }^{3}$, Junmeng

$$
\mathrm{Cai}^{1,2, *}
$$

${ }^{1}$ Biomass Energy Engineering Research Center, Key Laboratory of Urban Agriculture (South) Ministry of Agriculture, School of Agriculture and Biology, Shanghai Jiao Tong University, 800 Dongchuan Road, Shanghai 200240, People's Republic of China

${ }^{2}$ CAS Key Laboratory of Renewable Energy, Guangzhou Institute of Energy Conversion, Chinese Academy of Sciences, Guangzhou 510640, People's Republic of China

${ }^{3}$ European Bioenergy Research Institute, Aston University, Aston Triangle, Birmingham B4 7ET, United Kingdom

* Corresponding author: Junmeng Cai, Tel.: +0086-21-34206624; E-mail address: jmcai@sjtu.edu.cn; Website: http://biofuels.sjtu.edu.cn.

\section{Highlights}

Two theoretically simulated data sets for cellulose and xylan pyrolysis were 
analyzed by using the sum of square error surface tool.

$>$ The experimental kinetic data of cellulose pyrolysis was processed by using the logistic DAEM coupled with the pattern search method for parameter estimation.

Many parameter sets of the logistic DAEM can fit the data at different heating rates very well for both simulated and experimental processes, and the kinetic compensation effect between the parameters has been found.

\section{Abstract}

The kinetic compensation effect in the logistic distributed activation energy model (DAEM) for lignocellulosic biomass pyrolysis was investigated. The sum of square error (SSE) surface tool was used to analyze two theoretically simulated logistic DAEM processes for cellulose and xylan pyrolysis. The logistic DAEM coupled with the pattern search method for parameter estimation was used to analyze the experimental data of cellulose pyrolysis. The results showed that many parameter sets of the logistic DAEM could fit the data at different heating rates very well for both simulated and experimental processes, and a perfect linear relationship between the logarithm of the frequency factor and the mean value of the activation energy distribution was found. The parameters of the logistic DAEM can be estimated by coupling the optimization method and isoconversional kinetic methods. The results would be helpful for chemical kinetic analysis using DAEM. 
Key words: logistic distribution; distributed activation energy model (DAEM); kinetic compensation effect; biomass pyrolysis; pattern search method

\section{Graphical abstract}

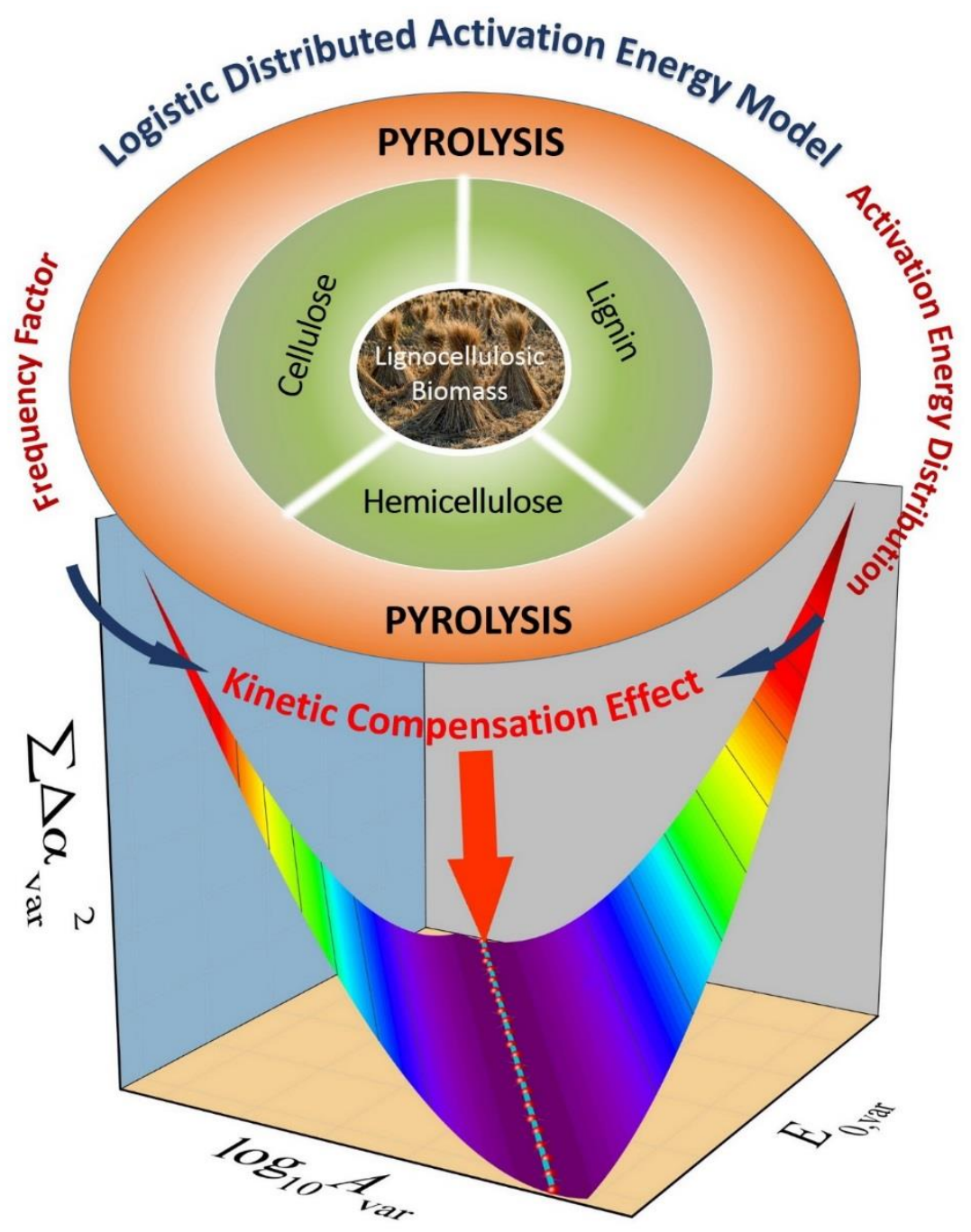




\section{Introduction}

The distributed activation energy model (DAEM) was originally developed by Pitt (1962) in order to analyze coal pyrolysis kinetics and later modified by Anthony (1976). Nowadays, it is considered as the most comprehensive model for describing the pyrolysis kinetics of lignocellulosic biomass and its components (Cai et al., 2014; Zhou et al., 2017). It assumes an infinite number of irreversible first order parallel reactions with different activation energies. The difference in activation energy is usually represented by a continuous distribution function $f(E)$. With some assumptions, the general form of the DAEM equation can be obtained (Cai et al., 2014):

$$
\alpha=\int_{0}^{\infty}\left\{1-\exp \left[-\int_{0}^{t} A \exp \left(-\frac{E}{R T}\right) \mathrm{d} t\right]\right\} g(E) \mathrm{d} E
$$

where $\alpha$ is the degree of conversion, $t$ is the time, $A$ is the frequency factor, $E$ is the activation energy, $R$ is the universal gas constant, $T$ is the absolute temperature, and $g(E)$ is the activation energy distribution.

In general, $g(E)$ is assumed to follow a Gaussian distribution because of its simplicity (Cai et al., 2014). However, the Gaussian distribution is not accurate enough for the initial and final stages of biomass pyrolysis. To describe reactions more accurately, the logistic distribution was proposed to present the activation energy distribution and applied to successfully describe cellulose pyrolysis kinetics (Cai et al., 2011a; Cai et al., 2011b):

$$
\alpha=\int_{0}^{\infty}\left[1-\exp \left(-\int_{0}^{t} A e^{-E / R T} \mathrm{~d} t\right)\right] \frac{b}{\sigma} \frac{e^{-b\left(E-E_{0}\right) / \sigma}}{\left[1+e^{-b\left(E-E_{0}\right) / \sigma}\right]^{2}} \mathrm{~d} E
$$


where $b=\frac{\pi}{\sqrt{3}}, E_{0}$ and $\sigma$ are the mean value and standard deviation of the logistic distribution, respectively. Latest developments of utilizing the logistic DAEM to fit the experimental kinetic data of biomass pyrolysis satisfactorily can be found in recent studies (Chen et al., 2017; Ghodke \& Mandapati, 2018; Kirtania \& Bhattacharya, 2015; Xiong et al., 2016).

Most laboratory experiments involving lignocellulosic pyrolysis kinetics are carried out under non-isothermal conditions at a linear heating rate $\beta=\mathrm{d} T / \mathrm{d} t$ (Mishra \& Mohanty, 2018). Under the linear heating program, Equation (2) becomes:

$$
\alpha=\int_{0}^{\infty}\left[1-\exp \left(-\int_{0}^{T} \frac{A}{\beta} e^{-E / R T} \mathrm{~d} T\right)\right] \frac{b}{\sigma} \frac{e^{-b\left(E-E_{0}\right) / \sigma}}{\left[1+e^{-b\left(E-E_{0}\right) / \sigma}\right]^{2}} \mathrm{~d} E
$$

There is no exact analytical solution of Equation (3). In our previous studies (Cai et al., 2014), the numerical calculation method was developed for processing the double integration in Equation (3).

In order to simplify the calculation, the frequency factor $(A)$ in the DAEM was usually fixed at a certain constant value (Güneş \& Güneş, 2008; Várhegyi et al., 2011). In our previous study (Cai \& Liu, 2008), a dependence of the frequency factor upon temperature $\left(A=A_{0} T^{n}\right)$ was proposed to improve the fit of the experimental kinetic data of biomass pyrolysis. The linear relationship between the logarithm of the frequency factor and the mean value of the activation energy distribution was introduced (Czajka et al., 2016; Huang et al., 2017):

$$
\ln A=a E_{0}+b
$$

where $a$ and $b$ are constants. The above dependence is also called as the kinetic 
compensation effect.

In widely used chemical kinetics, $\frac{\mathrm{d} \alpha}{\mathrm{d} t}=A e^{-E / R T} f(\alpha)$ (where $f(\alpha)$ is the reaction model), the kinetic compensation effect states a kind of linear relationship between the $\operatorname{logarithm}$ of the frequency factor $(\ln A)$ and the activation energy $(E)$. The kinetic compensation effect has been observed in numerous kinetic studies of solid-state reactions. Koga (1994) tried to find the intrinsic mechanism of the kinetic compensation effect and found that such dependence was caused by the properties of the general kinetic equation. Yip et al. (2011) studied the low-temperature oxidation of coal chars and found that the kinetic compensation effect was a result of the selective oxidation of coal chars with heterogeneous carbon structures according to FT-Raman spectroscopic analysis. Some purely mathematical reasons such as the random experimental and systematic errors also cause the compensation effect (Barrie, 2012a; Barrie, 2012b). According to an physical explanation, the compensation effect is caused by a correlation between the enthalpy change and the entropy change on going from the reagents to the transition state of the reaction (Perez-Benito, 2013). Parmon (2016) studied the kinetic compensation effect in complex stepwise reactions and found that the existence of the 'isokinetic' temperature in homogeneous systems caused the compensation effect.

There are many studies about the kinetic compensation effect in general chemical kinetics. However, the systematic analysis of the kinetic compensation effect in the DAEM is still missing. Therefore, the aim of this study is to analyze the intrinsic mechanism of the kinetic compensation effect in the DAEM by analyzing the 
theoretical simulated data and experimental data of biomass pyrolysis.

\section{Experiments and methods}

\subsection{Kinetic experiments of cellulose pyrolysis}

The experimental kinetic data of cellulose pyrolysis were obtained from Refs (Conesa et al., 1995; Conesa et al., 2004). In the experiments, the sample Whatman No. 6 paper was used as a representative material of cellulose. The kinetic experiments of cellulose pyrolysis were carried out on a thermogravimetric analyzer (Perkin-Elmer thermobalance model TGA7) under an inert atmosphere from room temperature to $450{ }^{\circ} \mathrm{C}$ at the heating rates of 5,25 and $50 \mathrm{~K} \mathrm{~min}^{-1}$. The experiments were repeated three times and their reproductivity was good. The experimental weight loss data obtained at each heating rate were averaged.

A biomass pyrolysis process involves the dehydration stage. The mechanism of water evaporation is different from that of the decomposition of biopolymer components. Therefore, the removal of the dehydration stage from the experimental weight loss data is the first thing for processing data. Kinetic analysis is commonly carried out based on the conversion degree. Therefore, the data needs to be transferred to the form of the conversion degree:

$$
\alpha(T)=\frac{w_{i}-w(T)}{w_{i}-w_{f}}
$$

where $w_{i}, w(T)$ and $w_{f}$ are the initial weight, the weight at the temperature $T$, and the 
final weight, respectively. Detailed information about processing data can be found in in our previous paper (Cai et al., 2018).

\subsection{Pattern search method for DAEM parameter estimation}

For fitting the experimental data of cellulose pyrolysis to the logistic DAEM, some estimation of the model parameters is needed. Then, the following objective function included the data obtained at different heating rates is defined for parameter estimation:

$$
\mathrm{OF}=\sum_{j=1}^{n_{h}} \sum_{i=1}^{n_{d, j}}\left(\alpha_{\mathrm{exp}, i j}-\alpha_{\mathrm{cal}, i j}\right)^{2}
$$

where the subscripts exp and cal represent the experimental data and the values calculated from the logistic DAEM, respectively, $i$ refers to the $i$ th data point, $j$ refers to the $j$ th heating rate, $n_{d, j}$ is the number of data points at the $j$ th heating rate and $n_{h}$ is the number of heating rates.

To obtain the parameters minimizing the objective function (6), a certain optimization method should be used. The function (6) has no obvious expression, and it is difficult to obtain its derivative information. In this study, the pattern search method is used. It can be used on functions that are not continuous or differentiable. The method can perform the poll and optional search step at every iteration. In addition, it can be used to perform robust local optimization more efficiently and effectively (Lewis et al., 2000).

For the implementation of the pattern search method, the Global Optimization Toolbox in MATLAB was used. 


\section{Results and discussion}

\subsection{Analysis of theoretically simulated data}

For obtaining the theoretically simulated data, the parameter values of two theoretical DAEM processes (as listed in Table 1), used for describing cellulose and xylan pyrolysis (Cai et al., 2013), were considered. Figure 1 shows the $\alpha-T$ curves of two simulated DAEM processes.

Table 1. Parameter values for simulated data for DAEM processes

\begin{tabular}{|c|c|c|c|c|}
\hline & $\begin{array}{c}\log _{10} A \\
\left(A \text { is expressed in } \mathrm{s}^{-1}\right)\end{array}$ & $\begin{array}{c}E_{0} \\
\left(\mathrm{~kJ} \mathrm{~mol}^{-1}\right)\end{array}$ & $\begin{array}{c}\sigma \\
\left(\mathrm{kJ} \mathrm{mol}{ }^{-}\right. \\
\left.{ }^{1}\right)\end{array}$ & $\begin{array}{c}\beta \\
\left(\mathrm{K} \mathrm{min}^{-}\right. \\
\left.{ }^{1}\right)\end{array}$ \\
\hline $\begin{array}{l}\text { Simulated } \\
\text { cellulose }\end{array}$ & 13.9 & 210.5 & 1.5 & 10 \\
\hline pyrolysis process & & & & \\
\hline $\begin{array}{l}\text { Simulated xylan } \\
\text { pyrolysis process }\end{array}$ & 12.7 & 178.3 & 5.8 & 10 \\
\hline
\end{tabular}




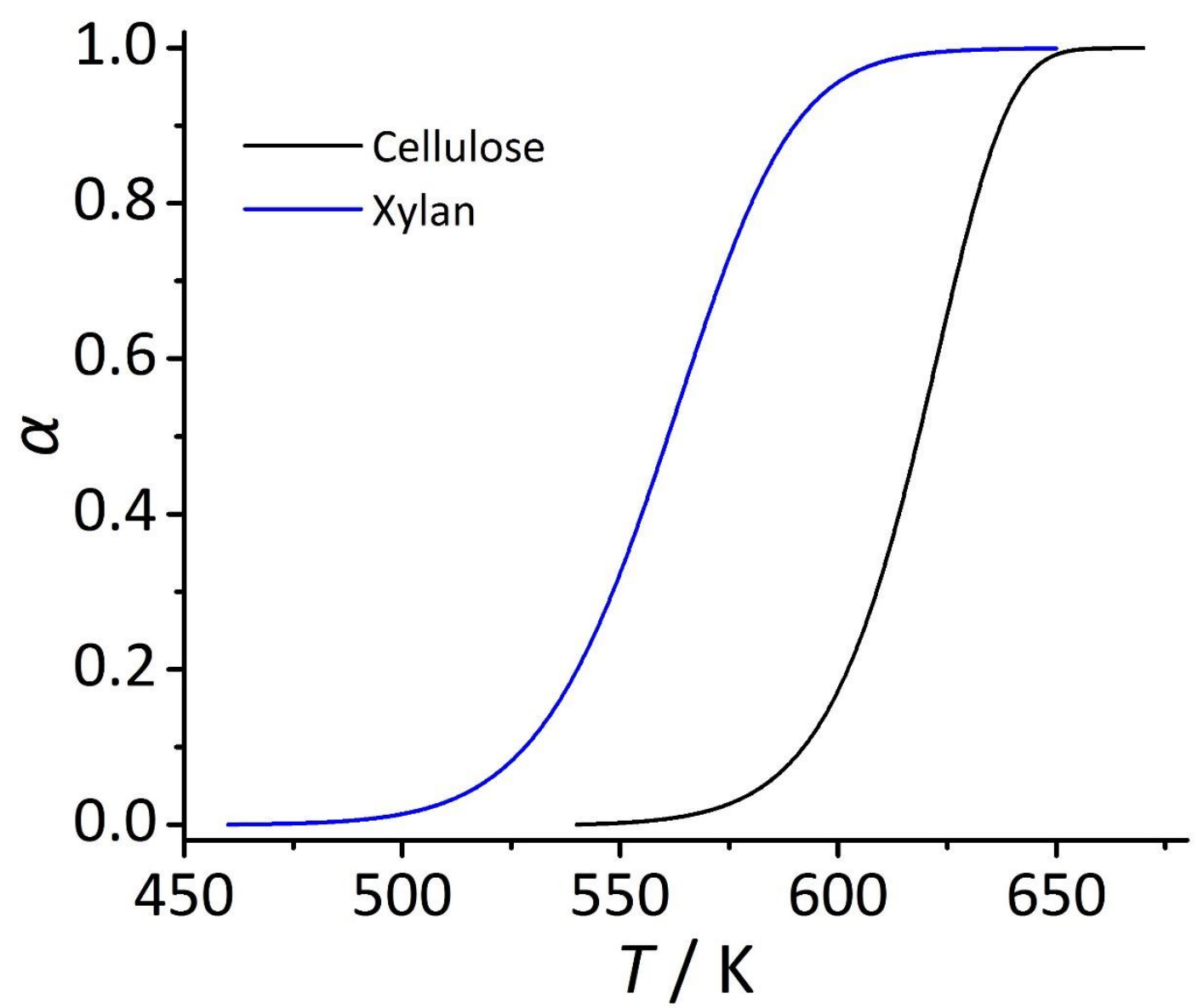

Figure 1. Conversion curves of two simulated DAEM processes for cellulose and xylan pyrolysis at $10 \mathrm{~K} \mathrm{~min}^{-1}$

According to Equation (3), it can be obtained that the conversion of a theoretically simulated DAEM process is a function of $T, A, E_{0}, \sigma$, and $\beta$. To investigate the effect of the variation of the parameters $A$ and $E_{0}$ on the simulated DAEM process, the sum of square error (SSE) surface tool is employed:

$$
\operatorname{SSE}\left(A_{\mathrm{var}}, E_{0, \mathrm{var}}\right)=\sum_{i=1}^{n_{d}}\left[\alpha_{\mathrm{var}}\left(T_{i}, A_{\mathrm{var}}, E_{0, \mathrm{var}}, \sigma_{\mathrm{sim}}, \beta_{\mathrm{sim}}\right)-\alpha_{\mathrm{sim}}\left(T_{i}, A_{\mathrm{sim}}, E_{0, \mathrm{sim}}, \sigma_{\mathrm{sim}}, \beta_{\mathrm{sim}}\right)\right]^{2}
$$

where $n_{d}$ is the number of data points, the subscript sim represents the values of the 
simulated process, while the subscript var is the varying values for the parameters $A$ and $E_{0}$. The SSE function is a measure of the discrepancy between the data calculated from the parameters $A_{\text {var }}$ and $E_{0, \text { var }}$ and the simulated data.

Figure 2 (a) and (b) show the SSE color map surfaces with the variation of $A_{\text {var }}$ and $E_{0, v a r}$ for simulated cellulose and xylan pyrolysis processes, respectively. It can be obtained that there is a long sharp valley in the SSE surface for both simulated processes. About 30 minimum SSE value points located in the valley are selected (also presented in Figure 2 (a) and (b)). As shown in Figure 2 (c) and (d), a perfect linear relationship between $\log _{10} A$ and $E_{0}$ of those points for both simulated cellulose and xylan pyrolysis processes is found. For convenience, those points will be called as compensation effect points in further analysis. Table 2 lists the linear regression results of $\log _{10} A$ versus $E_{0}$ for compensation effect points. From the above analyses, it can be obtained that the parameter sets corresponding to those compensation points can describe the simulated data at $10 \mathrm{~K} \mathrm{~min}^{-1}$ very well. 

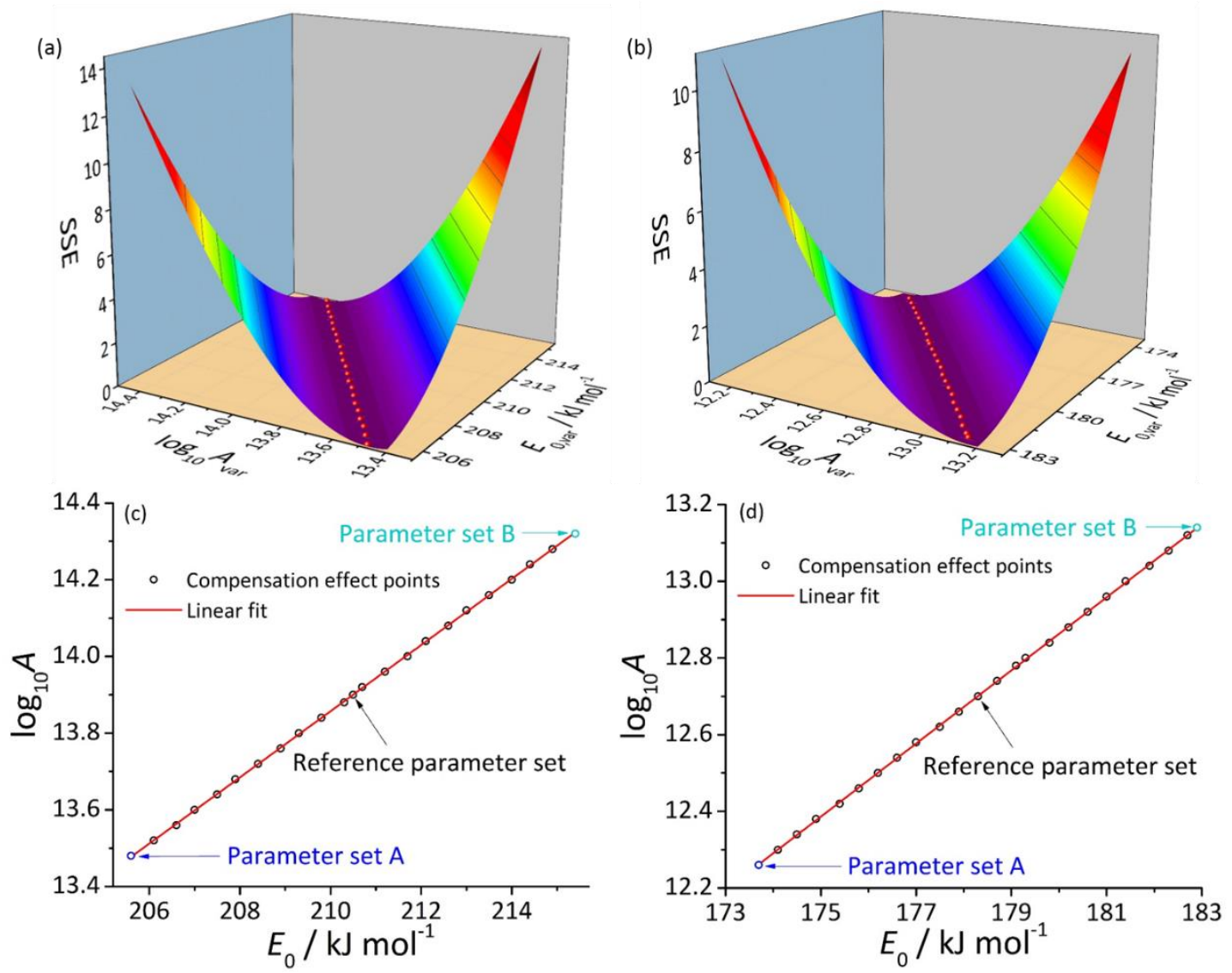

Figure 2. SSE surface for simulated (a) cellulose and (b) xylan pyrolysis processes, and linear fitting of compensation effect points for simulated (c) cellulose and (d) xylan pyrolysis processes at $10 \mathrm{~K} \mathrm{~min}^{-1}$

Table 2. Linear fit results of compensation effect points for simulated processes at 10 $\mathrm{K} \min ^{-1}$

\begin{tabular}{lccc}
\hline & $a$ & $b$ & $\mathrm{R}^{2}$ \\
\hline Simulated cellulose pyrolysis process & 0.08631 & -4.26819 & 0.99989 \\
Simulated xylan pyrolysis process & 0.09527 & -4.28093 & 0.99978 \\
\hline
\end{tabular}

In order to check whether the parameter sets corresponding to those compensation effect points can describe the data at other heating rates, the data at the heating rates of 
2.5, 5, 20 and $40 \mathrm{~K} \mathrm{~min}^{-1}$ for both simulated cellulose and xylan pyrolysis processes were considered. For this purpose, the parameter sets A and B (minimum and maximum $\log _{10} A$ and $E_{0}$ values, as shown in Table 3) were selected for the following analyses.

Table 3. Parameter values of parameter sets A and B for simulated cellulose and xylan pyrolysis processes

\begin{tabular}{|c|c|c|c|c|}
\hline & \multirow{3}{*}{ Parameter set } & \multirow{3}{*}{$\begin{array}{c}(A \text { is expressed in } \\
\left.\mathrm{s}^{-1}\right)\end{array}$} & \multirow{3}{*}{$\begin{array}{c}E_{0} \\
\left(\mathrm{~kJ} \mathrm{~mol}^{-}\right. \\
1)\end{array}$} & \multirow{3}{*}{$\begin{array}{c}\sigma \\
(\mathrm{kJ} \text { mol } \\
\\
\left.{ }^{1}\right)\end{array}$} \\
\hline & & & & \\
\hline & & & & \\
\hline Simulated cellulose pyrolysis & Parameter set A & 13.48 & 205.6 & 1.5 \\
\hline process & Parameter set B & 14.32 & 215.4 & 1.5 \\
\hline Simulated xylan pyrolysis & Parameter set A & 12.26 & 173.7 & 5.8 \\
\hline process & Parameter set B & 13.14 & 182.9 & 5.8 \\
\hline
\end{tabular}

Figure 3 shows the comparison among the $\alpha$-T curves with the reference parameter set, parameter set A and parameter set B at different heating rates for both simulated cellulose and xylan pyrolysis processes. From Figure 3, it can be obtained that the $\alpha$ $T$ curves with the parameter sets A and B are very close to the $\alpha-T$ curve with the reference parameter set at different heating rates. This indicates that the kinetic compensation effect existed in the parameters $\log _{10} A$ and $E_{0}$ is independent on the heating rate. 

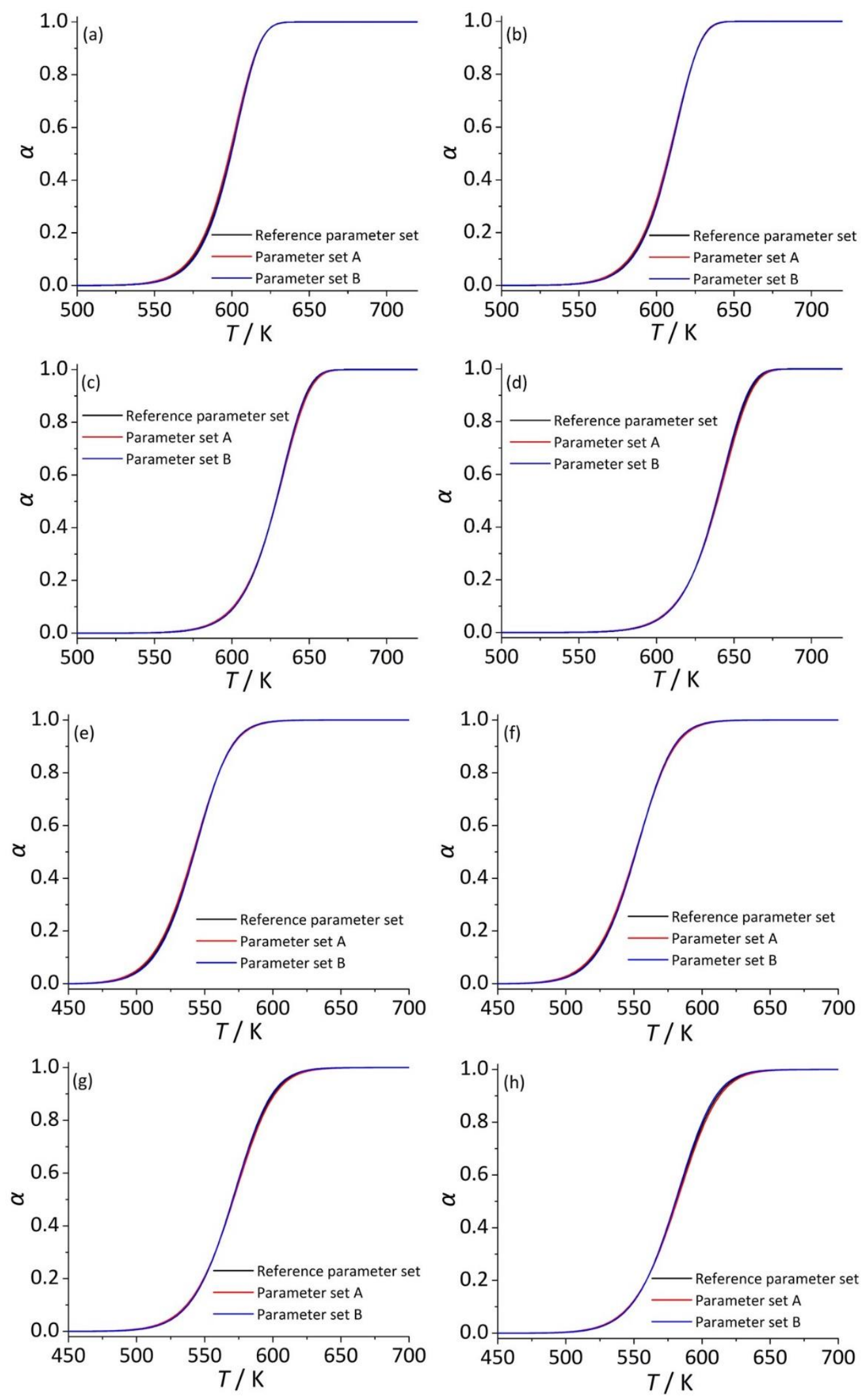

Figure 3. $\alpha-T$ curves with reference parameter set, parameter set $A$ and parameter set

B at (a) $2.5 \mathrm{~K} \mathrm{~min}^{-1}$; (b) $5 \mathrm{~K} \mathrm{~min}^{-1}$; (c) $20 \mathrm{~K} \mathrm{~min}^{-1}$; (d) $40 \mathrm{~K} \mathrm{~min}^{-1}$ for simulated 
cellulose pyrolysis process and (e) $2.5 \mathrm{~K} \mathrm{~min}^{-1}$; (f) $5 \mathrm{~K} \mathrm{~min}^{-1}$; (g) $20 \mathrm{~K} \mathrm{~min}^{-1}$; (h) 40 $\mathrm{K} \min ^{-1}$ for simulated xylan pyrolysis process

\subsection{Analysis of experimental data}

Figure 4 shows the objective function value, mesh size (which controls the degree of how to partition the searching space) and number of function evaluations per interval during the iterative optimization calculation process with the initial guess of the parameters: $\log _{10} A=13.9, E_{0}=210.5 \mathrm{~kJ} \mathrm{~mol}^{-1}$ and $\sigma=1.5 \mathrm{~kJ} \mathrm{~mol}^{-1}$, which are the same as the above simulated cellulose pyrolysis process. The corresponding optimal parameter values were established: $\log _{10} A=13.282, E_{0}=210.532 \mathrm{~kJ} \mathrm{~mol}^{-1}$ and $\sigma=0.768 \mathrm{~kJ} \mathrm{~mol}^{-1}$. As shown in Figure 5, the comparison between the experimental data and the curves calculated from the logistic DAEM with the optimal parameters $\left(\log _{10} A=13.282\right.$, $E_{0}=210.532 \mathrm{~kJ} \mathrm{~mol}^{-1}$ and $\sigma=0.768 \mathrm{~kJ} \mathrm{~mol}^{-1}$ ) at three heating rates indicates that the model can predict the experimental data quite satisfactorily. 

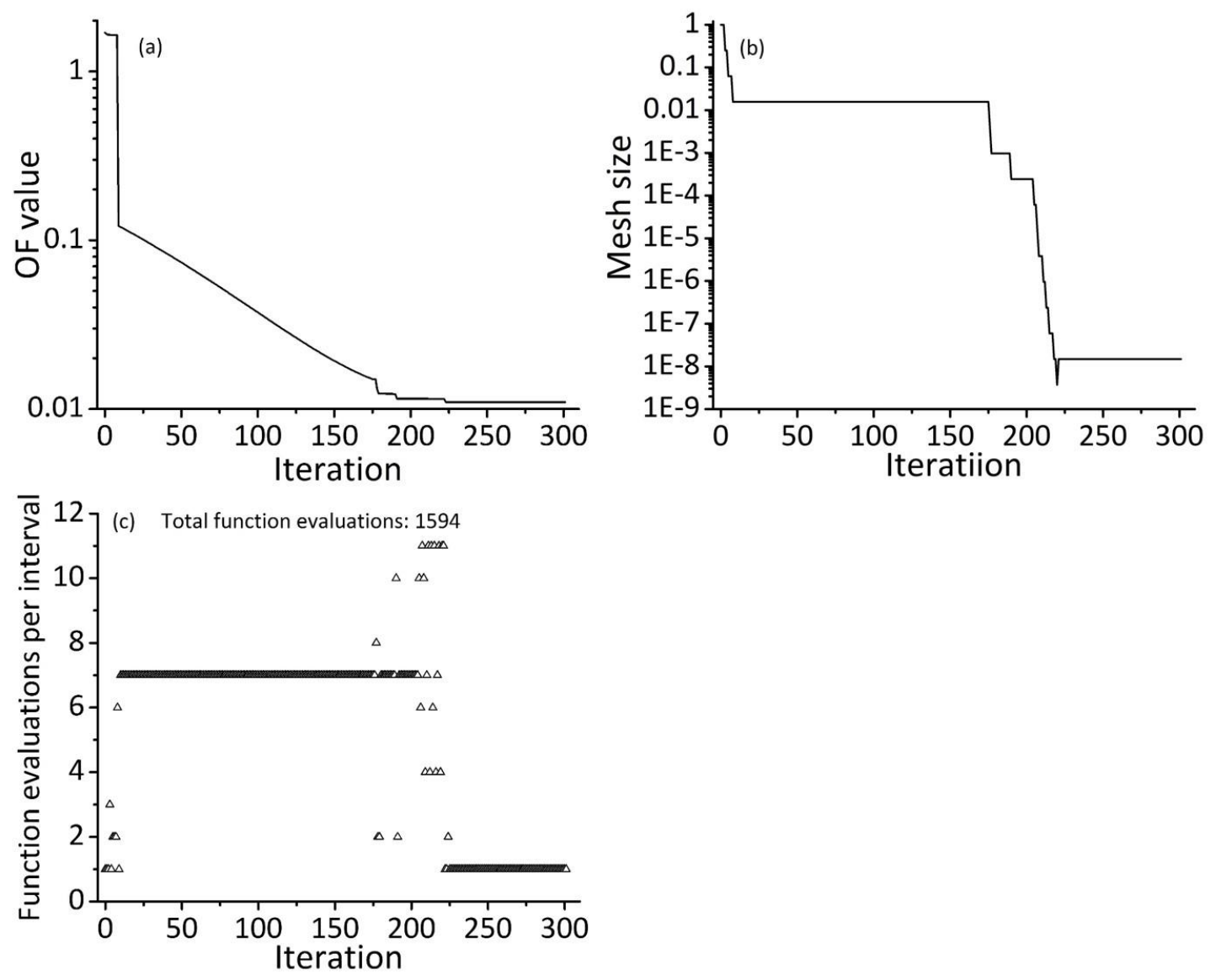

Figure 4. Objective function value, mesh size and function evaluations per interval during iterative optimization calculation process 


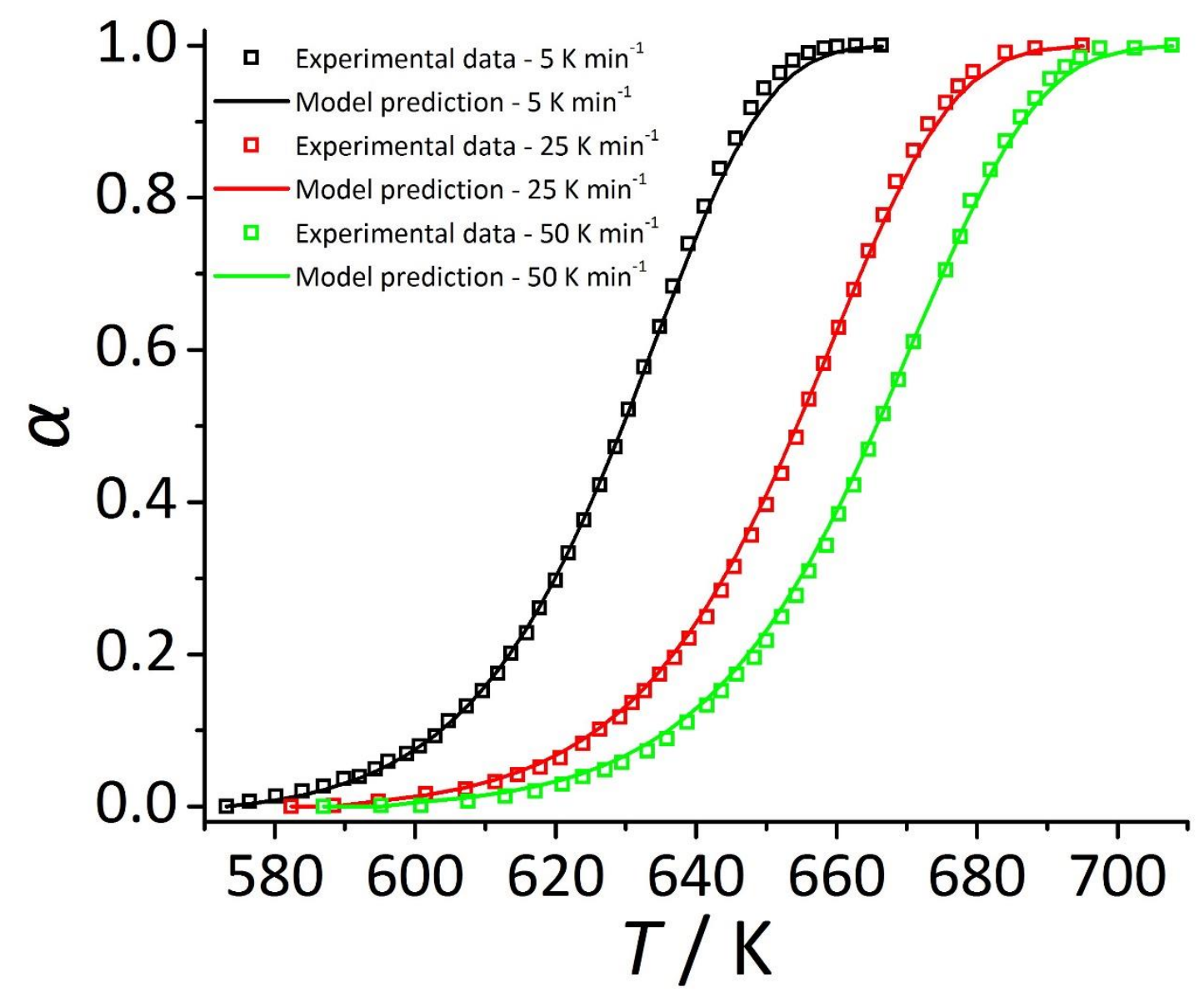

Figure 5. Comparison between experimental data and model prediction with one optimal parameter set: $\log _{10} A=13.282, E_{0}=210.532 \mathrm{~kJ} \mathrm{~mol}^{-1}$ and $\sigma=0.768 \mathrm{~kJ} \mathrm{~mol}^{-1}$

It has been found from many calculation results that different initial guesses of the parameters lead to different optimal parameter sets. To investigate the relationship between $\log _{10} A$ and $E_{0}$ for various optimal parameter sets, the value of $\sigma$ is fixed at $0.768 \mathrm{~kJ} \mathrm{~mol}^{-1}$. Various optimal parameter sets, the corresponding objective function values and $\mathrm{R}^{2}$ values of the experimental data and model predictions at different heating rates are obtained and listed in Table 4. It can be obtained that the logistic DAEM with different optimal parameter sets leads to the perfect fit of the experimental data at all heating rates. 
Table 4. Several parameter sets optimized with different initial guesses of $\log _{10} A$ and $E_{0}$ and fixed value of $\sigma$

\begin{tabular}{cccccccc}
\hline & & & & & \multicolumn{3}{c}{$\mathrm{R}^{2}$} \\
\cline { 5 - 7 } No. & $\log 10 A\left(A\right.$ is expressed in $\left.\mathrm{s}^{-1}\right)$ & $E_{0} / \mathrm{kJ} \mathrm{mol}^{-1}$ & $\begin{array}{c}\sigma / \mathrm{kJ} \\
\mathrm{mol}^{-1}\end{array}$ & OF value & $\begin{array}{c}5 \mathrm{~K} \\
\mathrm{~min}^{-1}\end{array}$ & $\begin{array}{c}25 \mathrm{~K} \\
\mathrm{~min}^{-1}\end{array}$ & $\begin{array}{c}50 \mathrm{~K} \\
\mathrm{inin}^{-1}\end{array}$ \\
\hline 1 & & & & & & & \\
& 12.840 & 205.143 & 0.768 & $1.79907 \times 10^{-2}$ & 0.99970 & 0.99971 & 0.99981 \\
2 & 12.924 & 206.173 & 0.768 & $1.58004 \times 10^{-2}$ & 0.99975 & 0.99973 & 0.99982 \\
3 & 13.021 & 207.350 & 0.768 & $1.37153 \times 10^{-2}$ & 0.99980 & 0.99976 & 0.99984 \\
4 & 13.081 & 208.081 & 0.768 & $1.26633 \times 10^{-2}$ & 0.99982 & 0.99977 & 0.99985 \\
5 & 13.196 & 209.483 & 0.768 & $1.11344 \times 10^{-2}$ & 0.99986 & 0.99979 & 0.99985 \\
6 & 13.282 & 210.532 & 0.768 & $1.04152 \times 10^{-2}$ & 0.99988 & 0.99981 & 0.99985 \\
7 & 13.402 & 212.001 & 0.768 & $1.02001 \times 10^{-2}$ & 0.99989 & 0.99981 & 0.99985 \\
8 & 13.563 & 213.968 & 0.768 & $1.04903 \times 10^{-2}$ & 0.99991 & 0.99985 & 0.99985 \\
9 & 13.697 & 215.598 & 0.768 & $1.17857 \times 10^{-2}$ & 0.99991 & 0.99986 & 0.99983 \\
\hline
\end{tabular}

Considering optimal parameter sets listed in Table 4, the curve of $\log _{10} A$ versus $E_{0}$ is presented in Figure 6, where a perfect linear relationship between $\log _{10} A$ and $E_{0}$ can be observed $\left(\mathrm{R}^{2}=1.00000\right): \log _{10} A=0.08196 E_{0}-3.97279\left(A\right.$ is expressed in $\mathrm{s}^{-1}, E_{0}$ is expressed in $\mathrm{kJ} \mathrm{mol}^{-1}$ ). 


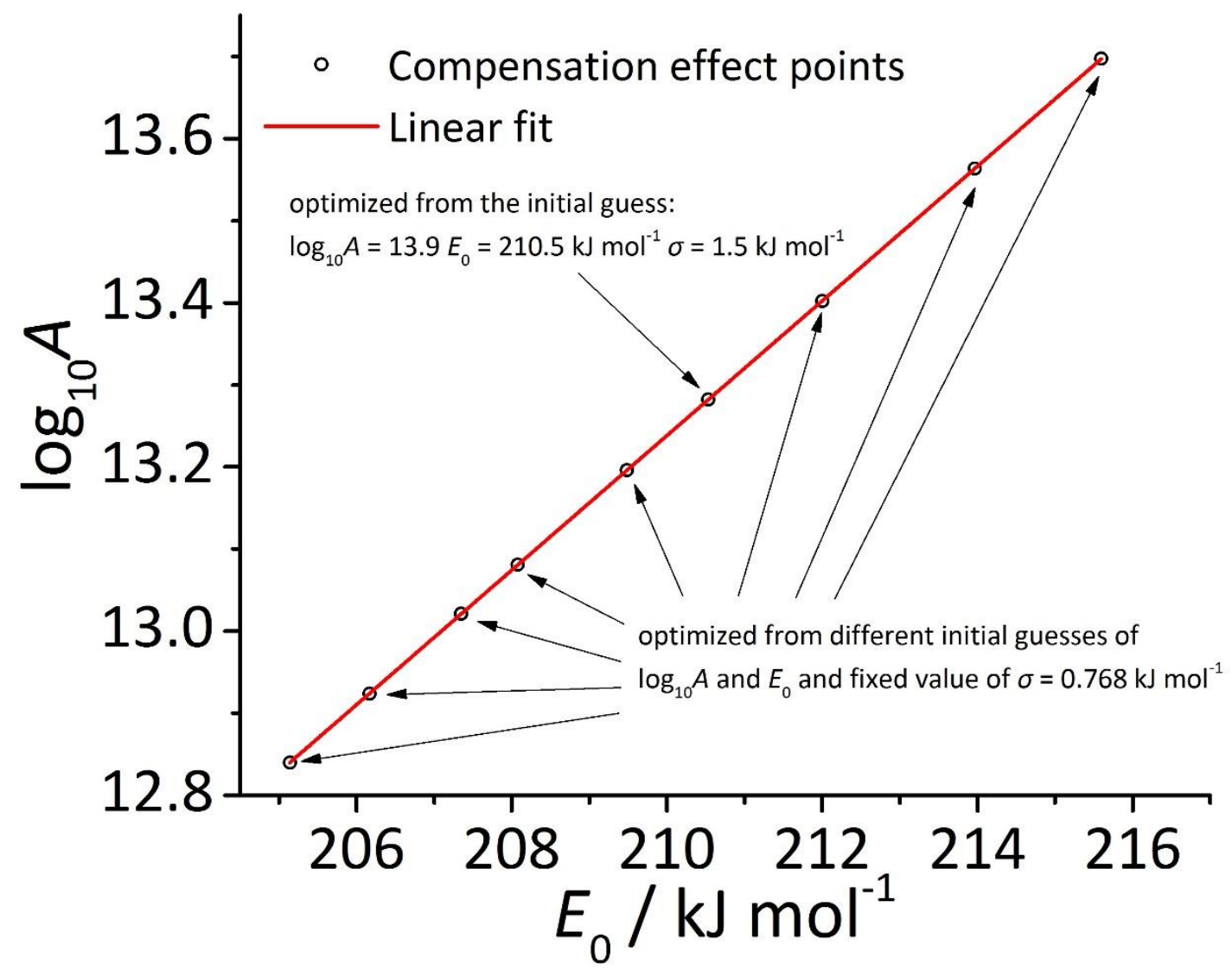

Figure 6. Linear fit of compensation effect points for experimental data of cellulose pyrolysis

\section{Implications for biomass pyrolysis kinetic analysis}

According to the above analyses, different parameter sets can fit a logistic DAEM process equally well (from the statistical point of view) for both simulated and experimental data for biomass pyrolysis. The values of $A$ and $E_{0}$ differ and the linear relationship between $\log _{10} A$ and $E_{0}$ for different parameter sets is found. If the activation energy distribution could be pre-determined by means of isoconversional methods $(\mathrm{Wu}$ et al., 2013), the frequency factor in the logistic DAEM would be determined according 
to the kinetic compensation effect; or if the frequency factor could be pre-determined, the activation energy distribution would be obtained by means of the combination of a certain optimization method and the kinetic compensation effect.

\section{Conclusions}

The kinetic compensation effect in the logistic DAEM was comprehensively studied through processing theoretically simulated cellulose and xylan pyrolysis data and experimental data of cellulose pyrolysis by means of the SSE surface tool and pattern search method. The results showed that there existed many parameter sets fitting the simulated data equally well and that the linear relationship between the logarithm of the frequency factor and the mean value of the activation energy distribution (kinetic compensation effect in DAEM) independent upon the heating rate was found. The results would be helpful for chemical kinetic analysis using DAEM.

\section{Acknowledgments}

Financial support from CAS Key Laboratory of Renewable Energy (No. Y807k91001) and Global Challenges Research Fund (GCRF) Networking Grant ('Pyrolysis of Municipal Organic Waste for Renewable Road Construction Materials') is greatly acknowledged. 


\section{References}

1. Anthony, D.B. 1976. Coal devolatilization and hydrogasification. AIChE Journal, 22(4), 625-656.

2. Barrie, P.J. 2012a. The mathematical origins of the kinetic compensation effect: 1. The effect of random experimental errors. Physical Chemistry Chemical Physics, 14(1), 318-326.

3. Barrie, P.J. 2012b. The mathematical origins of the kinetic compensation effect: 2. The effect of systematic errors. Physical Chemistry Chemical Physics, 14(1), 327336.

4. Cai, J., Jin, C., Yang, S., Chen, Y. 2011a. Logistic distributed activation energy model--Part 1: Derivation and numerical parametric study. Bioresource Technology, 102(2), 1556-1561.

5. Cai, J., Liu, R. 2008. New distributed activation energy model: Numerical solution and application to pyrolysis kinetics of some types of biomass. Bioresource Technology, 99(8), 2795-2799.

6. Cai, J., Wu, W., Liu, R. 2014. An overview of distributed activation energy model and its application in the pyrolysis of lignocellulosic biomass. Renewable and Sustainable Energy Reviews, 36, 236-246.

7. Cai, J., Wu, W., Liu, R., Huber, G.W. 2013. A distributed activation energy model for the pyrolysis of lignocellulosic biomass. Green Chemistry, 15(5), 1331-1340.

8. Cai, J., Xu, D., Dong, Z., Yu, X., Yang, Y., Banks, S.W., Bridgwater, A.V. 2018. 
Processing thermogravimetric analysis data for isoconversional kinetic analysis of lignocellulosic biomass pyrolysis: Case study of corn stalk. Renewable and Sustainable Energy Reviews, 82, 2705-2715.

9. Cai, J., Yang, S., Li, T. 2011b. Logistic distributed activation energy model--part 2: application to cellulose pyrolysis. Bioresource Technology, 102(3), 3642-3644.

10. Chen, Z., Lei, J., Li, Y., Su, X., Hu, Z., Guo, D. 2017. Studies on thermokinetic of Chlorella pyrenoidosa devolatilization via different models. Bioresource Technology, 244(Pt 1), 320-327.

11. Conesa, J.A., Caballero, J., Marcilla, A., Font, R. 1995. Analysis of different kinetic models in the dynamic pyrolysis of cellulose. Thermochimica Acta, 254, 175-192.

12. Conesa, J.A., Caballero, J.A., Reyes-Labarta, J.A. 2004. Artificial neural network for modelling thermal decompositions. Journal of Analytical and Applied Pyrolysis, 71(1), 343-352.

13. Czajka, K., Kisiela, A., Moroń, W., Ferens, W., Rybak, W. 2016. Pyrolysis of solid fuels: Thermochemical behaviour, kinetics and compensation effect. Fuel Processing Technology, 142, 42-53.

14. Güneş, M., Güneş, S. 2008. Distributed activation energy model parameters of some Turkish coals. Energy Sources, 30(16), 1460-1472.

15. Ghodke, P., Mandapati, R.N. 2018. Kinetic modeling of Indian rice husk pyrolysis. International Journal of Chemical Reactor Engineering, 16(2), Paper No. 20170048.

16. Huang, Y.W., Chen, M.Q., Li, Y. 2017. An innovative evaluation method for kinetic 
parameters in distributed activation energy model and its application in thermochemical process of solid fuels. Thermochimica Acta, $\mathbf{6 5 5}, 42-51$.

17. Kirtania, K., Bhattacharya, S. 2015. Coupling of a distributed activation energy model with particle simulation for entrained flow pyrolysis of biomass. Fuel Processing Technology, 137, 131-138.

18. Koga, N. 1994. A review of the mutual dependence of Arrhenius parameters evaluated by the thermoanalytical study of solid-state reactions: The kinetic compensation effect. Thermochimica Acta, 244(C), 1-20.

19. Lewis, R.M., Torczon, V., Trosset, M.W. 2000. Direct search methods: then and now. Journal of Computational and Applied Mathematics, 124(1), 191-207.

20. Mishra, R.K., Mohanty, K. 2018. Pyrolysis kinetics and thermal behavior of waste sawdust biomass using thermogravimetric analysis. Bioresource Technology, 251, 63-74.

21. Parmon, V.N. 2016. Kinetic compensation effects: a long term mystery and the reality. A simple kinetic consideration. Reaction Kinetics, Mechanisms and Catalysis, 118(1), 165-178.

22. Perez-Benito, J.F. 2013. Some tentative explanations for the enthalpy-entropy compensation effect in chemical kinetics: from experimental errors to the Hinshelwood-like model. Monatshefte für Chemie - Chemical Monthly, 144(1), 49-58.

23. Pitt, G.J. 1962. The kinetic of the evolution of volatile products from coal. Fuel, 41, 267-274. 
24. Várhegyi, G., Bobály, B., Jakab, E., Chen, H. 2011. Thermogravimetric study of biomass prolysis kinetics. A distributed activation energy model with prediction tests. Energy \& Fuels, 25(1), 24-32.

25. Wu, W., Cai, J., Liu, R. 2013. Isoconversional kinetic analysis of distributed activation energy model processes for pyrolysis of solid fuels. Industrial \& Engineering Chemistry Research, 52(40), 14376-14383.

26. Xiong, Q., Zhang, J., Xu, F., Wiggins, G., Stuart Daw, C. 2016. Coupling DAEM and CFD for simulating biomass fast pyrolysis in fluidized beds. Journal of Analytical and Applied Pyrolysis, 117, 176-181.

27. Yip, K., Ng, E., Li, C.-Z., Hayashi, J.-I., Wu, H. 2011. A mechanistic study on kinetic compensation effect during low-temperature oxidation of coal chars. Proceedings of the Combustion Institute, 33(2), 1755-1762.

28. Zhou, B., Zhou, J., Zhang, Q. 2017. Research on pyrolysis behavior of Camellia sinensis branches via the Discrete Distributed Activation Energy Model. Bioresource Technology, 241, 113-119. 